\title{
Derecho animal en Cataluña. Las pautas de Francia
}

\author{
Marita Giménez-Candela \\ Catedrática \\ Directora del ICALP y del Máster en Derecho Animal UAB \\ ORCID: 0000-0002-0755-5928 \\ SCOPUS ID: 57218923647
}

Cita recomendada. Giménez-Candela, M., Derecho Animal en Cataluña. Las pautas de Francia, dA. Derecho Animal (Forum of Animal Law Studies) 12/3 (2021). - DOI https://doi.org/10.5565/rev/da.600

\section{Resumen}

En tres puntos sumarios (según la tradición cartesiana tan querida en Francia), se exponen algunos aspectos en que Cataluña se ha posicionado como una sociedad en la que la protección animal es prioritaria, tal como reflejan las iniciativas jurídicas y el impulso positivo que la sociedad catalana ha manifestado a lo largo de los últimos años. Cataluña, en el conjunto del estado español, ha demostrado siempre un espíritu pionero en situar a los animales en el lugar que jurídica, política y socialmente les corresponde como seres sentientes y que está reconocido por la legislación de bienestar animal de la Unión Europea. En este sentido, la afinidad entre Francia y Cataluña, con un mismo espíritu de respeto por los animales, debe ser puesta de relieve y servir de punto de partida para una mas estrecha colaboración.

Palabras clave: Derecho Animal; seres sentientes; estatuto jurídico de los animales; enseñanza del Derecho Animal; respeto por los animales; Cataluña; Francia.

\section{Abstract - Animal Law in Catalonia. The French model}

In three summary points (according to the so loved Cartesian tradition), this piece presents certain aspects of how Catalonia has positioned itself as a society that animal protection, as reflected by the legal initiatives and the positive push of society in recent years. Catalonia, in conjunction with the Spanish state, has always demonstrated a pioneering spirit for placing animals in the position that legally, politically and socially corresponds to them as sentient beings and as recognised by the animal welfare legislation of the European Union. In this sense, the affinity between France and Catalonia, with a shared spirit of respect for animals, must be highlighted and serve as a starting point for closer collaboration.

Keywords: Animal law; sentient beings; legal status of animals; teaching animal law; respect for animals; Catalonia; France. 


\section{Sumario $^{1}$}

1. El estatuto jurídico de los animales en el Código civil catalán y en el Código civil español

2. La protección animal en Catalunya: actuaciones pioneras

2.1.1 Prohibición de las corridas de toros

2.1.2 Animales urbanos

\subsection{Perros en el Metro}

2.1.2.1.2 Pienso anticonceptivo para jabalíes

2.1.2.1.3 Colonias de gatos

2.1.2.1.4 Palomas

2.1.2.1.5 Circos

2.1.2.1.6 Zoo y delfinario

2.1.3 Creación de Oficinas de Bienestar Animal en los Ayuntamientos catalanes

2.1.4 Creación de Comisiones de Protección animal en los Colegios de Abogados catalanes

3. La enseñanza universitaria (interdisciplinar) del Derecho Animal en Cataluña

3.1.1 El Máster en Derecho Animal y Sociedad

3.1.2 La optativa "Derecho y Bienestar Animal"

3.1.3 El ICALP (International Center for Animal Law and Policy)

\section{El estatuto jurídico de los animales en el Código civil catalán y en el Código civil español}

La primera cuestión que conviene brevemente puntualizar es que Cataluña, como territorio histórico que es, tiene un derecho propio cuya identidad y vigencia está reconocido por la Constitución española de $1978^{2}$, ello hace que Cataluña tenga un Código civil propio que ${ }^{3}$, en tema de estatuto jurídico de los animales, se ha diferenciado durante muchos años del Código civil de España. Lo expongo a continuación de manera sucinta.

La mayoría de los códigos continentales otorgan a los animales la categoría de cosas en propiedad, como herencia de la configuración romana de la propiedad sobre aquellas cosas (esclavos y animales) mas importantes para una economía agraria como la romana ${ }^{4}$. El Código Napoleón ${ }^{5}$, recogió -como no podía ser de otra forma- esta configuración romana que ha pervivido, sin excesivas críticas por parte del Derecho (pero sí del pensamiento filosófico, francés por cierto ${ }^{6}$ ), hasta la segunda mitad del S. XX tomando como epicentro algunos países de Centroeuropa. En concreto, en 1988 el ABGB de Austria cambia la categoría jurídica de los

\footnotetext{
*Agradezco a mis compañeros en el ICALP y la secretaría de redacción de la revista, Raffaela Cersosimo, Oliver Wookey y Silvia Zanini, por su ayuda y aportaciones durante la redacción de este artículo.

${ }^{1}$ Este texto recoge, revisado y adaptado, el contenido de mi intervención en el "Debate Animal en línea", celebrado en París el 15 de abril de 2021, en la sede de la Delegación del Gobierno de Cataluña en Francia.

2 Vid. por todos, CORCUERA ATIENZA, J., La constitucionalización de los derechos históricos. Fueros y Autonomía, en Revista española de Derecho Constitucional 11 (1984) 9ss. http://www.jstor.org/stable/44202898

3 VAQUER ALOY, A., El derecho civil catalán: presente y futuro, en Revista jurídica de Navarra 46 (2008) 69ss. http://hdl.handle.net/10459.1/66188

${ }^{4}$ GIMÉNEZ-CANDELA, T, Derecho Privado Romano (Valencia 2020) 165, 166, 168; CAPOGROSSI COLOGNESI, L., Das Eigentum in Rom vom Ende der patriarchalischen Ordnung bis zur Blütezeit der Sklavereiordnung, en Scritti scelti (Roma 2010) 299323; RATHBONE, D., Rural Economy and Society in Roman Italy (Cambridge University Press 2004); FINLEY, M.I., Ancient Economy (California University Press 1999); DUNCAN-JONES, R., Structure \& Scale in the Roman Economy (Cambridge University Press 1990). Sobre estatuto jurídico de los animales, de forma actualizada, cfr. GIMÉNEZ-CANDELA, M., Transición animal en España (Valencia 2020) 159-257.

${ }^{5}$ El Código civil de los franceses de 1804 se llamó oficialmente Código Napoleón (Code Napoléon) en 1807 (Décret du 3 septembre 1807).

${ }^{6}$ Vid. por todos, una excelente síntesis de CHAPOUTHIER, G., Le respect de l'animal dans ses racines historiques: de l'animal object à l'animal sensible, en Revue trimestrielle Droit Animal, Éthique \& Sciences 110 (2008) https://www.fondation-droitanimal.org/documentation/respect-de-lanimal-objet-a-lanimal-sensible/
} 
animales de cosas a la de "no-cosas", haciéndose eco de una fuerte demanda social, que denotaba un "giro animal" que empezó a tomar fuerza en algunos países europeos, que secundaron este mismo cambio. Me refiero a Alemania, que adopta en el BGB la misma denominación de "no-cosas"8 para los animales (1989) y Suiza que hace lo mismo ${ }^{9}$ un año más tarde $(1990)^{10}$.

Lo mismo hizo Cataluña en el año 2006, en el tratado de la propiedad de su propio Código civil que, de forma original y pionera en el ámbito hispano, declaró a los animales como "no-cosas"11, de acuerdo con la justificación de modernización del texto del Código civil catalán, iniciada con la Ley 13/1984, de 20 de marzo, de reforma de la Compilación del derecho civil de $1960^{12}$ a la que, de forma precisa, se refiere el preámbulo de la Ley 5/2006, de 10 de mayo $^{13}$, del libro quinto del Código civil de Cataluña, respecto al contenido del titulo I del mencionado Código, en los siguientes términos:

"El título I está configurado por algunos artículos, de carácter introductorio y general, sobre el régimen jurídico de los bienes, cuyo concepto se toma en un sentido amplio, de modo que incluye los derechos y, de acuerdo con la tradición jurídica catalana más reciente, establece que los animales no tienen la consideración de cosas y están bajo la protección de las leyes”.

Vale la pena destacar que, en dicho preámbulo, el legislador catalán precisa que "el régimen jurídico de los bienes (...), de acuerdo con la tradición jurídica catalana más reciente, establece que los animales no tienen la consideración de cosas". Es decir, que dicha declaración tímidamente descosificadora de los animales (que, sin embargo significó un gran paso), para el legislador catalán tiene sus raíces en la "tradición jurídica catalana más reciente". No comentaré aquí, por no ser el lugar, la alusión claramente distorsionada a una "tradición jurídica reciente", pues, en sí mismo, la asociación de los términos "tradición" y "reciente" parecen un oxímoron ${ }^{14}$. Pero, quizá el legislador lo que pretendía decir es que Cataluña ha sido una Comunidad Autónoma avanzada respecto de las restantes, en tema de protección de los animales, por la temprana iniciativa de configurar legalmente un régimen jurídico animal, de carácter europeo, por medio de la promulgación de la ley autonómica de protección de los animales ya en $1988^{15}$, que fue pionera en su género en el ámbito autonómico y que ha sido la fuente de inspiración para la correspondiente legislación promulgada por las restantes Comunidades Autónomas.

Volviendo al Título I, De los bienes, el redactado del art. 511-1, 3, tiene el siguiente tenor:

\footnotetext{
7 §285a ABGB: "Tiere sind keine Sachen; sie werden durch besondere Gesetze geschützt. Die für Sachen geltenden Vorschriften sind auf Tiere nur insoweit anzuwenden, als keine abweichenden Regelungen bestehen." ("Los animales no son cosas; están protegidos por leyes especiales. Las disposiciones referidas a las cosas se aplican a los animales, si no hay una previsión diferente.”- Traducción no oficial).

$8 \S 90$ a BGB: "[Tiere] Tiere sind keine Sachen. Sie werden durch besondere Gesetze geschützt. Auf sie sind die für Sachen geltenden Vorschriften entsprechend anzuwenden, soweit nicht etwas anderes bestimmt ist." ("Los animales no son cosas. Están protegidos mediante leyes especiales. A ellos se les deben aplicar las correspondientes disposiciones válidas para las cosas, siempre que no esté prevista otra cosa." - Traducción no oficial). https://www.gesetze-im-internet.de/bgb/_90a.html

9 Art. 641a ZGB: "Tiere sind keine Sachen" ("Los animales no son cosas" - Traducción no oficial). https://www.fedlex.admin.ch/eli/cc/24/233_245_233/de

${ }^{10}$ Vid. de forma detallada, GIMÉNEZ-CANDELA, M., Transición animal en España (cit.) 231ss.

${ }^{11}$ Art. 511-1.3 CCCat.: "Los animales, que no se consideran cosas, están bajo la protección especial de las leyes. Solo se les aplican las reglas de los bienes en lo que permite su naturaleza". https://www.boe.es/eli/es-ct/1/2006/05/10/5/con

${ }^{12}$ Ley 13/1984, de 20 de marzo, de reforma de la Compilación del derecho civil de $1960 \mathrm{https}: / /$ www.boe.es/eli/es-ct/1/1984/03/20/13

${ }^{13}$ Ley 5/2006, de 10 de mayo, del libro quinto del Código civil de Cataluña, relativo a los derechos reales «DOGC» núm. 4640, de 24/05/2006, «BOE» núm. 148, de 22/06/2006, BOE-A-2006-11130. El Preámbulo alude a las necesidades sociales y a los avances de la ciencia jurídica, en clara alusión a los debates acerca de la naturaleza de no-cosas de los animales, que en aquel momento eran de actualidad en los regímenes jurídicos europeos, en los siguientes términos que transcribo: "Este proceso, iniciado con la Ley 13/1984, de 20 de marzo, de reforma de la Compilación del derecho civil de 1960, ha continuado a lo largo de varias legislaturas. Los hitos más relevantes han sido la aprobación del Código de sucesiones, por la Ley 40/1991, de 30 de diciembre; del Código de familia, por la Ley 9/1998, y de la Ley 10/1998, de uniones estables de pareja, ambas de 15 de julio, y de la Ley 29/2002, de 30 de dicie mbre, primera ley del Código civil de Cataluña, la cual establece su estructura y elaboración en forma de código abierto, que es preciso construir a partir de un proceso continuado y que en el futuro puede adaptarse de forma flexible a las necesidades sociales y a los avances de la ciencia jurídica de cada momento".

${ }^{14}$ Una acepción generalmente aceptada sobre tradición jurídica es la que afirma que "una tradición es una creencia o un comportamiento (costumbre popular) que se transmite dentro de un grupo o sociedad con un significado simbólico o una significación especial con orígenes en el pasado". Vid. por todos, MERRYMAN, J.H., The civil Law Tradition. An Introduction to the legal Systems of Europe and Latin America (Stanford University Press. $3^{\text {rd }}$ Ed. 2007)

${ }^{15}$ Ley 3/1988, de 4 de marzo, de protección de los animales https://www.boe.es/eli/es-ct/1/1988/03/04/3, reformada sucesivamente en 2003, 2006 y, por último en 2008, Decreto Legislativo 2/2008, de 15 de abril, por el que se aprueba el Texto refundido de la Ley de protección de los animales https://www.boe.es/buscar/pdf/2008/DOGC-f-2008-90016-consolidado.pdf
} 
"Los animales, que no se consideran cosas, están bajo la protección especial de las leyes. Solo se les aplican las reglas de los bienes en lo que permite su naturaleza".

La modificación del Código catalán, presenta, pues, los mismos condicionantes, que los establecidos en los respectivos artículos del ABGB (Austria), BGB (Alemania) y Suiza (ZGB), para referirse al cambio de estatuto jurídico de los animales, pues usa el equivalente en castellano, al giro alemán "nicht Sachen" (=no son cosas) al emplear la expresión: "que no se consideran cosas", sin atreverse el legislador, en el 2006, a hacer una aseveración más coherente con el carácter de seres sintientes ("sentient beings") de los animales, como tres años más tarde dejaría taxativamente establecido el art $13 \mathrm{TFUE}^{16}$, que es mandatorio para todos los Estados Miembros y ha tenido una enorme influencia, al establecer la sentiencia como estándar para calificar jurídicamente a los animales, todo y que el término sentiencia, aún no estando reconocido por la RAE, resulta de común uso incluso en el lenguaje forense ${ }^{17}$.

Sin embargo, no fue hasta 2015 que Francia introdujo un cambio decisivo, diría revolucionario ${ }^{18}$, en la categorización jurídica de los animales, pues los convirtió en "seres vivos dotados de sensibilidad"19 lo que los aparta claramente de la posible asimilación con las cosas (también las inertes). Francia abrió con dicha reforma una puerta de reflexión profunda, muy importante ${ }^{20}$, pues la mencionada modificación es acorde con la condición de los animales como seres sentientes ("sentient beings"), tal como ha demostrado la Ciencia del Bienestar Animal y está reflejado en la legislación europea de Bienestar Animal, como en 2009 dejó plasmado el art. 13 del TFUE.

El Código civil español, sin embargo, ha estado anclado hasta el día 2 de diciembre de 2021 a la consideración de los animales como cosas en propiedad, después de un largo recorrido de intentos de reforma ${ }^{21}$, que afortunadamente ha culminado con la aprobación de esta importante modificación ${ }^{22}$. Este cambio supone un paso adelante muy necesario. Sin él nuestro ordenamiento hubiera seguido chocando con dos concepciones sobre los animales claramente antitéticas: la consideración de los mismos como $\operatorname{cosas}^{23}$ y la del reconocimiento de los animales como lo que son: seres sentientes ${ }^{24}$, lo que, por cierto, ya había estado

\footnotetext{
16 TFUE, art. 13: https://eur-lex.europa.eu/legal-content/ES/TXT/?uri=CELEX:12016E013: “Al formular y aplicar las políticas de la Unión en materia de agricultura, pesca, transporte, mercado interior, investigación y desarrollo tecnológico y espacio, la Unión y los Estados miembros tendrán plenamente en cuenta las exigencias en materia de bienestar de los animales como seres sensibles, respetando al mismo tiempo las disposiciones legales o administrativas y las costumbres de los Estados miembros relativas, en particular, a ritos religiosos, tradiciones culturales y patrimonio regional."

${ }^{17}$ Sobre sentiencia, vid. GIMÉNEZ-CANDELA, M., Transición animal en España (cit.) 213ss.

${ }^{18}$ GIMÉNEZ-CANDELA, T., Una nueva Revolución Francesa: la modernización del Code civil, dA. Derecho Animal (Forum of Animal Law Studies) 6/1 (2015) 1-2. https://doi.org/10.5565/rev/da.270. El llamado Amendement Glavany por el nombre de uno de los diputados que presentó la propuesta de reforma, hizo uso de un proyecto general de "modernización" de la legislación francesa, para introducir el cambio de estatuto jurídico de los animales de cosas a "êtres vivants doués de sensibilité": Proyecto de Ley de modernización y simplificación del Derecho en los ámbitos de la Justicia y Asuntos de Interior (No. 1808) https://www.assembleenationale.fr/14/amendements/1808/AN/59.asp. Vid. con visión pionera, desde 2009, MARGUÉNAUD, J.P. Avant-Propos, en RSDA 1 (2009) 7ss.; ANTOINE, S., Le projet de réforme du droit des biens, en RSDA 1 (2009) 11ss; MARGUÉNAUD, J.P., L'entrée en vigueur de "l'amendement Glavany": un grand pas de plus vers la personnalité juridique des animaux, RSDA 2 (2014), 15ss.

19 Art. 515-14 C.civ.: "Les animaux sont des êtres vivants doués de sensibilité. Sous réserve des lois qui les protègent, les animaux sont soumis au régime des biens." ("Los animales son seres vivos dotados de sensibilidad. Si no hay leyes que les protegen, a los animales se aplica el régimen jurídico de los bienes" - Traducción no oficial).

https://www.legifrance.gouv.fr/codes/article_lc/LEGIARTI000030250342/

${ }^{20}$ Hago aquí un reconocimiento a la importante labor llevada a cabo por el Prof. Jean Pierre Marguenaud y sus discípulos de la Université de Limoges, para dotar de fundamento doctrinal al cambio de estatuto jurídico de los animales en el Code civil francés. Por todos, vid. MARGUÉNAUD, J.-P., LEROY, J., BOISSEAU-SOWINSKI, L., Code de l'Animal (Paris 2018); MARGUÉNAUD, J.P., BURGAT, F., LEROY, J., Le droit animalier (Paris 2016); MARGUÉNAUD, J.-P., L'animal en droit privé (Paris 1992); Revue Semestrielle de Droit Animalier: https://idedh.edu.umontpellier.fr/publications/; Sobre las actividades académicas del grupo de Limoges, vid. también, GIMÉNEZ-CANDELA, M., CERSOSIMO, R., La enseñanza del derecho animal (Valencia 2021) 60, 70, 116120.

${ }^{21}$ CERDEIRA BRAVO DE MANSILLA, G., ¿Un nuevo Derecho civil para los animales?: Elogio (no exento de enmiendas) a la nueva Proposición de Ley sobre el régimen jurídico de los animales, en España, dA. Derecho Animal (Forum of Animal Law Studies) 12/2 (2021) 39-53. DOI https://doi.org/10.5565/rev/da.573

${ }^{22}$ GIMÉNEZ-CANDELA, M., Estatuto jurídico de los animales en el Código civil. La esperada descosificación animal, dA. Derecho Animal (Forum of Animal Law Studies) 12/2 (2021). - DOI https://doi.org/10.5565/rev/da.582

${ }^{23}$ Las principales normas se centraban en el Libro Segundo "De los bienes, propiedades y sus modificaciones" del CC. https://boe.es/buscar/act.php?id=BOE-A-1889-4763 . Sobre el tema, vid. por todos, ROGEL VIDE, C., Los animales en el Código Civil (Madrid 2017); GIMÉNEZ-CANDELA, M., Transición animal en España (cit.) 159-257.

${ }^{24}$ La modificación del Código Civil ayudará también a armonizar la legislación sobre los derechos de los animales que tienen las diferentes Comunidades Autónomas. Así, mientras Cataluña aprobó en 2003 que los animales son seres dotados de "sensibilidad psíquica" y por tanto merecedores de unos derechos propios, el resto de las comunidades no tenían en aquel entonces ninguna legislación específica al respecto.
} 
propuesto tanto en alguna legislación autonómica como la de Canarias desde $2015^{25}$, incluido en la Ley de Protección animal de Castilla la Mancha ${ }^{26}$. Y, por supuesto, también incluido en toda la legislación del Bienestar Animal que España ha traspuesto a su ordenamiento, como Estado miembro que es de la Unión Europea $^{27}$.

\section{La protección animal en Catalunya: actuaciones pioneras}

Cataluña, ya antes de la reforma referida de 2006, ha sido y es una sociedad muy sensible a la protección animal. La mentalidad abierta y la apertura a los cambios que la sociedad demanda, podría decirse que resulta connatural al espíritu de la ciudadanía catalana. Por eso, no es de extrañar que en Cataluña, como ocurre también en Francia, algunas reformas e iniciativas a favor de los animales hayan cristalizado mucho antes que en otros territorios de España. Me referiré, de forma sucinta, a algunas de ellas:

\subsection{Prohibición de las corridas de toros}

Las corridas de toros se prohibieron en territorio catalán el 28 de julio de 2010, gracias a una Iniciativa Legislativa Popular (ILP), tras de un intenso periodo de recogida de firmas que evidenció un gran apoyo popular a la prohibición. Aunque en 2016 el Tribunal Constitucional español anuló la abolición de las corridas de toros, hay que decir que, desde 2010, no se han vuelto a celebrar corridas en las plazas catalanas ${ }^{28}$.

\subsection{Animales urbanos ${ }^{29}$}

\subsubsection{Perros en el Metro}

Barcelona fue la primera ciudad de España que permitió en 2014 que los perros viajaran en el Metro. Fue un cambio pequeño, si se quiere, pero importante porque señalaba que los perros se integraban sin dificultad en una nueva sociedad mas abierta, inclusiva y tolerante. La reforma la propuso el ICALP, el Centro de Investigación que dirijo en la UAB, que hizo los estudios de viabilidad y contribuyó a la reforma de los estatutos de los transportes municipales (TMB) ${ }^{30}$.

Como anécdota debo decir que los primeros trenes de España que permitieron viajar a los perros fueron, precisamente, los Ferrocarriles de la Generalitat de Cataluña (FGC).

\subsubsection{Pienso anticonceptivo para jabalís}

Barcelona es una ciudad rodeada de un parque natural boscoso que es la sierra de Collserola y toda Cataluña es un territorio de montañas y bosques en las comarcas de interior. Como secuela del cambio climático y de la ocupación de los antiguos límites naturales del bosque, en los últimos años se ha producido un gran crecimiento de las poblaciones de jabalís, que cada vez están más cerca de los núcleos urbanos donde buscan la comida que no tienen en sus antiguos territorios. En Cataluña se ha desarrollado un proyecto pionero para contener el crecimiento de las colonias de jabalís, evitar las posibles molestias a los ciudadanos y, al mismo tiempo, actuar con arreglo a parámetros éticos y de respeto por el bienestar de estos animales. En lugar de permitir cazarlos, ponerles trampas u otras soluciones cruentas, en Cataluña se les suministra piensos anticonceptivos, lo que progresivamente está siendo una solución respetuosa y eficaz ${ }^{31}$.

\footnotetext{
${ }^{25}$ De acuerdo a la Resolución aprobada por el Parlamento de Canarias del 16 de septiembre de 2015, la Consejería de Presidencia, Justicia e Igualdad inició el estudio para la revisión de la vigente Ley 8/1991, de 30 de abril, de protección de los animales, pero los cambios en la composición del gobierno autonómico, frustraron dicha iniciativa.

${ }^{26}$ Ley 7/2020, de 31 de agosto, de Bienestar, Protección y Defensa de los Animales de Castilla-La Mancha https://www.boe.es/eli/escm/1/2020/08/31/7 ; Vid. especialmente, GISIE, L., Comentario jurídico de la Ley 7/2020, de 31 de agosto, de Bienestar, Protección y Defensa de los Animales de Castilla-La Mancha. [2020/6154] - Diario Oficial de Castilla-La Mancha de 07-09-2020, en dA. Derecho Animal (Forum of Animal Law Studies) 12/1 (2021). - DOI https://doi.org/10.5565/rev/da.556

${ }^{27}$ De este cambio, que abre una era de cambios jurídicos en España, me ocuparé con detalle en breve. Baste decir ahora que, desde 2006 y, de forma reconocida y documentada desde 2009, he dedicado los últimos años a promover este cambio desde el ámbito académico, por considerar que es, precisamente el Código civil la norma que vertebra la consideración jurídica de los animales y el núcleo sustantivo donde se asienta el tratamiento de los animales en un ordenamiento jurídico como el nuestro, dentro del Civil Law. ${ }^{28}$ GIMÉNEZ-CANDELA, M., Transición animal en España (cit.) 139-141.

${ }^{29}$ La normativa vigente en Cataluña sobre animales urbanos, contextualizada con la vigente y relativa legislación estatal, se encuentra más adelante (Anexo I).
}

${ }^{30}$ GIMÉNEZ-CANDELA, M., Transición animal en España (cit.) 292-295.

${ }^{31}$ Vid. Anexo I. 


\subsubsection{Colonias de gatos}

La ley de protección animal catalana desde $2008^{32}$ estableció la competencia de los Ayuntamientos en el cuidado de los animales perdidos, abandonados y en el control de las colonias de gatos callejeros. Gracias a una eficaz batería de medidas de aplicación, dado que en los refugios de animales de Cataluña está prohibida la eutanasia, las colonias de gatos callejeros están acogidas al método de captura/esterilización/retorno lo que ha permitido una convivencia armónica de gatos ferales y de ciudadanos. Hay que señalar el alto nivel de la investigación en materia de comportamiento felino de alguna Universidades catalanas y la dedicación pionera de algunas asociaciones de protección animal dedicadas a los gatos ${ }^{33}$.

\subsubsection{Palomas}

Las palomas son un atractivo de muchas plazas y núcleos urbanos de Cataluña, pero también su proliferación genera muchos gastos de conservación del patrimonio urbano, por lo que en Barcelona se inició en 2017 lo que se conoce como un control poblacional ético para reducir su cuantía en un 70\% en el 2022. El Colegio de Abogados de Barcelona ha abanderado esta iniciativa que cuenta con la colaboración del Servicio de Ecopatología (SEFAS) y Fauna Salvaje de la UAB ${ }^{34}$.

\subsubsection{Circos}

El Parlamento de Cataluña prohibió el 22 de julio de 2015 las actuaciones de los circos con animales silvestres en todo el territorio catalán. Esta fue una iniciativa pionera en España, que desencadenó una cadena de prohibiciones acogidas por muchas ciudades de España. La reforma la propuso y gestionó la plataforma "Catalunya Lliure d'animals al circ" (CLAC), impulsada por la asociación animalista LIBERA y la Fundación Franz Weber, que ya en 2012 había suscrito un pacto de legislatura con los principales partidos políticos ${ }^{35}$.

\subsubsection{Zoo y delfinario}

En julio de 2020 los tres últimos delfines del delfinario de Barcelona fueron trasladados a un delfinario en Grecia, después de que se gestionara, sin éxito, el traslado a un santuario de estos inteligentes y bellos animales. De esta forma el Ayuntamiento de Barcelona cumplía su promesa de hacer de Barcelona una ciudad libre de cetáceos en cautividad, aunque este traslado, insatisfactorio para muchos animalistas, ha sido severamente criticado. Ya en 1994 la orca Ulises fue trasladada a un santuario de San Diego en California $(\mathrm{USA})^{36}$.

El Zoo de Barcelona ha sido muy cuestionado por las asociaciones animalistas de Barcelona, durante muchos años. En 2018 el Ayuntamiento aprobó la modificación de la ordenanza municipal de protección animal, para prohibir la reproducción en el Zoo de las especies que no pudieran ser liberadas, que tenía como finalidad cerrar el Zoo. Sin embargo, los cambios políticos en la gestión municipal han paralizado esta modificación y el Zoo de Barcelona, con nuevas inversiones, continúa su andadura a día de hoy ${ }^{37}$.

\subsection{Creación de Oficinas de Bienestar Animal en los Ayuntamientos catalanes}

La Oficina de Protección Animal (OPAB) del Ayuntamiento de Barcelona se creó en 2009. Fue, en términos absolutos, el primer servicio municipal creado en España con el objeto de velar por la protección y el bienestar animal en el ámbito municipal. Desde esa fecha, la OPAB ha desarrollado una labor incansable, que ha dado acogida o impulso a todas las iniciativas que he detallado anteriormente, destinadas a favorecer un trato digno de los animales en el ámbito municipal ${ }^{38}$. Después de muchos años, sin que el ejemplo de Barcelona tuviera seguidores en otros municipios de España, a día de hoy ya hay capitales de provincia, como

\footnotetext{
${ }^{32}$ Decreto Legislativo 2/2008 del 15 de abril, por el cual se aprueba el Texto refundido de la Ley de Protección de los Animales art.16.1

${ }^{33}$ Vid. por todos, DUFAU, A., Estatuto Jurídico del Gato Callejero en España, Francia y Reino Unido (Valencia 2017). Vid. también Anexo I.

${ }^{34}$ Vid. https://sct.uab.cat/sefas/en: Vid. también Anexo I.

${ }^{35}$ GIMÉNEZ-CANDELA, M., Transición animal en España (cit.) 151

${ }^{36}$ Vid.: https://www.zoobarcelona.cat/es/ulisses

${ }^{37}$ Vid.: https://www.zoobarcelona.cat/es/sostenibilidad

${ }^{38}$ Vid.: https://ajuntament.barcelona.cat/benestaranimal/es/oficina-de-proteccion-de-los-animales-opab
} 
Zaragoza, que cuentan con el mismo servicio ${ }^{39}$. Por lo demás muchos municipios catalanes, como Sabadell y Terrassa, han creado oficinas de protección animal y han promovido una decidida mejora de las ordenanzas municipales de protección animal ${ }^{40}$.

\subsection{Creación de Comisiones de Protección animal en los Colegios de Abogados catalanes}

Los Colegios de Abogados de Cataluña han creado en los últimos años las Comisiones de Protección Animal, o de Derechos de los Animales, en un ejercicio de concienciación de los profesionales del Derecho, cuyo punto de partida se sitúa en 2002, cuando el ICAB (Colegio de Abogados de Barcelona) toma la iniciativa. Es realmente digno de ser resaltado, que lo que, hace unos años, era una singularidad de Barcelona y después de Cataluña, es hoy un movimiento que se ha expandido por todos los Colegios de Abogados de España. Estas Comisiones prestan un gran servicio a la protección animal, pues son el cauce por donde los ciudadanos canalizan sus deseos de que ningún maltrato animal quede sin sanción y de que el Derecho recoja en la legislación, las mejoras que demanda el reconocimiento de los animales como seres sentientes ("sentient beings") $)^{41}$.

\section{La enseñanza universitaria (interdisciplinar) del Derecho Animal en Catalunya}

La Enseñanza del Derecho Animal es, a mi juicio, la clave para que los derechos de los animales formen parte del horizonte ético-jurídico de los ciudadanos. Enseñar a respetar a los animales y a mostrar a la ciudadanía los instrumentos para que el ejercicio de los mismos sea eficaz, se admite, hoy en día, que es un desarrollo inclusivo de los derechos de todos los seres vivientes y por tanto en la misma línea de protección a los vulnerables, que ha permitido que las grandes causas de la humanidad (voto femenino, trabajo infantil, seguridad de los trabajadores, etc.) hayan encontrado su lugar, de forma progresiva, dentro del ordenamiento jurídico.

Desde estas premisas y con el deseo de que la experiencia de enseñanza del Derecho Animal que voy a exponer, sea una batería de ideas para poder ser aplicada en muchos ámbitos de la enseñanza -también aquí en Francia-, voy a pasar a explicar algunas de las formas de enseñanza del Derecho Animal en el ámbito universitario, que vengo practicando en Cataluña y desde Cataluña. Mi pensamiento se podría concretar en esta breve máxima: "Saber más, para trabajar mejor por los animales"42.

\subsection{La optativa "Derecho y Bienestar Animal"}

Las Facultades de Derecho en Europa son ordinariamente conservadoras y tardan en incorporar a los planes de enseñanza nuevas enseñanzas, que la sociedad requiere. Un conjunto de circunstancias favorables permitió que, ya en 2007, se diera vía libre, en el plan de estudios oficial de la Facultat de Derecho de la Universitat Autònoma de Barcelona, a una materia optativa que se denomina "Derecho y Bienestar Animal". En esa fecha el término "Derecho Animal" provocaba rechazo, cuando no bromas y escepticismo entre mis colegas. Pero los estudiantes, desde el primer día, acogieron con entusiasmo una materia que a día de hoy es la optativa más demandada por los estudiantes de Derecho de la UAB. Somos la única Facultad de Europa que incluye el Derecho Animal en el plan de estudios de una Facultad jurídica y me gustaría que el ejemplo fuera seguido por muchas otras Facultades, en Francia, desde luego, y en toda Europa ${ }^{43}$.

\subsection{El Máster en Derecho Animal y Sociedad}

El Master en Derecho Animal y sociedad empezó su andadura en el 2009, bajo la forma de un curso de

\footnotetext{
${ }^{39}$ Vid.: https://www.zaragoza.es/ciudad/centros/detalle_Centro?id=10585

40 JIMÉNEZ-LÓPEZ, I, LORENTE RIVERA, C.J., Comentario sobre la nueva ordenanza municipal de protección, tenencia y bienestar animal de Sabadell, en dA. Derecho Animal (Forum of Animal Law Studies) 9/4 (2018) 129-137. https://doi.org/10.5565/rev/da.380

${ }^{41}$ Por todos, vid.el ICAB: https://www.icab.cat/es/colegio/comisiones-y-secciones/comite/Comision-de-Proteccion-de-los-Derechosde-los-Animales/ ; el ICASBD: https://www.icasbd.org/ca/serveis/defensa-dels-drets-dels-animals; el ICATER: https://icater.org/elcol-legi/comissions/\#1509457102357-181ec2b1-dd7b

${ }^{42}$ Sobre los programas de Derecho Animal en España y Francia, vid. GIMÉNEZ-CANDELA, T., Enseñanza del Derecho Animal en España, dA. Derecho Animal (Forum of Animal Law Studies) 6/4 (2015) 1-4. https://doi.org/10.5565/rev/da.263; MARGUÉNAUD, J.-P., La création d'un premier diplôme universitaire de droit animalier en France, en RSDA 1 (2016) 15-24; GIMÉNEZ-CANDELA, M., CERSOSIMO, R., La enseñanza del derecho animal (cit.) 102-113, 116-120.

${ }^{43}$ De forma más detallada, vid. GIMÉNEZ-CANDELA, M., CERSOSIMO, R., La enseñanza del derecho animal (cit.) 102, $327-241$.
} 
Postgrado. Tengo que decir que no sabía si el primer día de clase iba a estar yo sola. No fue así y el curso siguiente, 2010-2011, empezó ya el Máster en Derecho Animal (presencial) con la forma y los contenidos que actualmente tiene. Es decir, este año se está celebrando la $10^{\mathrm{a}}$ edición presencial y al mismo tiempo, la $7^{\mathrm{a}}$ edición online. Cuando empezamos, entendí que la formación en Derecho Animal tenía necesariamente que alcanzar a los profesionales que no pudieran desplazarse a Barcelona y pudieran aprovechar la enseñanza a través de las nuevas tecnologías. La experiencia, aunque intensa, ha sido y es altamente satisfactoria. Es mérito de un cuerpo docente, experto en la materia que enseñan, y de unos alumnos que desempeñan su trabajo, lo aprendido en el Máster, con gran competencia y solidez. Sólo añadiré, que nuestro hecho diferencial es que el Master es marcadamente interdisciplinar e internacional. Una fórmula de éxito, pues no se puede amar a los animales y aprender a protegerlos de forma eficaz, si no se dispone de una visión amplia e inclusiva ${ }^{44}$.

\subsection{EI ICALP (International Center for Animal Law and Policy)}

No concibo la enseñanza sin investigación. Son, en mi opinión, un tándem inescindible que se retroalimenta. Por esa razón, ya desde el 2010 promoví la creación de un grupo de investigación interdisciplinar denominado "Grupo ADS" (Animales-Derecho Sociedad) que luego originó el actual ICALP (International Center for Animal Law and Policy). En el ICALP conviven especialistas de distintas ramas del Derecho, las Humanidades, el Mediambiente y las Ciencias del Bienestar Animal. Fuimos el primer grupo de investigación de estas características en España. Se han realizado tesis doctorales, se han formado jóvenes investigadores, se participa en proyectos de investigación nacionales y europeos, se han organizado seminarios, congresos, webinars. En definitiva, todo lo que supone investigar y comunicar los resultados. Por eso, tanto la web jurídica derechoanimalo.info y la revista dA (Forum of Animal Law Studies) que está indexada en SCOPUS, son vehículos de las tareas que los investigadores del Centro llevan a cabo ${ }^{45}$.

\section{REFERENCIAS}

\section{BIBLIOGRAFÍA}

- ANTOINE, S., Le projet de réforme du droit des biens, en RSDA 1 (2009) 11ss

- CAPOGROSSI COLOGNESI, L., Das Eigentum in Rom vom Ende der patriarchalischen Ordnung bis zur Blütezeit der Sklavereiordnung, en Scritti scelti (Roma 2010) 299-323

- CERDEIRA BRAVO DE MANSILLA, G., ¿Un nuevo Derecho civil para los animales?: Elogio (no exento de enmiendas) a la nueva Proposición de Ley sobre el régimen jurídico de los animales, en España, dA. Derecho Animal (Forum of Animal Law Studies) 12/2 (2021) 39-53. DOI https://doi.org/10.5565/rev/da.573

- CHAPOUTHIER, G., Le respect de l'animal dans ses racines historiques: de l'animal object à l'animal sensible, en Revue trimestrielle Droit Animal, Éthique \& Sciences 110 (2008) https://www.fondation-droit-animal.org/documentation/respect-de-lanimal-objet-a-lanimalsensible/

- CORCUERA ATIENZA, J., La constitucionalización de los derecho históricos. Fueros y Autonomía, en Revista española de Derecho Constitucional 11 (1984) 9ss. http://www.jstor.org/stable/44202898

- DUFAU, A., Estatuto Jurídico del Gato Callejero en España, Francia y Reino Unido (Valencia 2017)

- DUNCAN-JONES, R., Structure \& Scale in the Roman Economy (Cambridge University Press 1990)

- FINLEY, M.I., Ancient Economy (California University Press 1999)

- GIMÉNEZ-CANDELA, M., Transición animal en España (Valencia 2020)

- GIMÉNEZ-CANDELA, T., Derecho Privado Romano (Valencia 2020)

\footnotetext{
${ }^{44}$ De forma más detallada, vid. GIMÉNEZ-CANDELA, T, El Máster en Derecho Animal y Sociedad (UAB), dA. Derecho Animal (Forum of Animal Law Studies) 7/4 (2016) 1-3. https://doi.org/10.5565/rev/da.320; GIMÉNEZ-CANDELA, T, Máster Online en Derecho Animal y Sociedad ( $1^{\text {a }}$ ed.), en dA. Derecho Animal (Forum of Animal Law Studies) 6/2 (2015) 1-2. https://doi.org/10.5565/rev/da.269; GIMÉNEZ-CANDELA, M., CERSOSIMO, R., La enseñanza del derecho animal (cit.) 103-113, $143,145,154$.

${ }^{45}$ De forma más detallada, vid. GIMÉNEZ-CANDELA, T., ICALP - International Centre for Animal Law and Policy, en dA. Derecho Animal (Forum of Animal Law Studies) 7/2 (2016) 1-5. https://doi.org/10.5565/rev/da.258; GIMÉNEZ-CANDELA, M., CERSOSIMO, R., La enseñanza del derecho animal (cit.) 58, 90, 109-113, 114.
} 
- GIMÉNEZ-CANDELA, M., Estatuto jurídico de los animales en el Código civil. La esperada descosificación animal, dA. Derecho Animal (Forum of Animal Law Studies) 12/2 (2021). - DOI https://doi.org/10.5565/rev/da.582

- GIMÉNEZ-CANDELA, T., ICALP - International Centre for Animal Law and Policy, en dA. Derecho Animal (Forum of Animal Law Studies) 7/2 (2016) 1-5. https://doi.org/10.5565/rev/da.258

- GIMÉNEZ-CANDELA, T, El Máster en Derecho Animal y Sociedad (UAB), dA. Derecho Animal (Forum of Animal Law Studies) 7/4 (2016) 1-3. https://doi.org/10.5565/rev/da.320

- GIMÉNEZ-CANDELA, T., Una nueva Revolución Francesa: la modernización del Code civil, dA. Derecho Animal (Forum of Animal Law Studies) 6/1 (2015) 1-2. https://doi.org/10.5565/rev/da.270.

- GIMÉNEZ-CANDELA, T, Máster Online en Derecho Animal y Sociedad (1 ${ }^{\mathrm{a}}$ ed.), en dA. Derecho Animal (Forum of Animal Law Studies) 6/2 (2015) 1-2. https://doi.org/10.5565/rev/da.269

- GIMÉNEZ-CANDELA, T., Enseñanza del Derecho Animal en España, dA. Derecho Animal (Forum of Animal Law Studies) 6/4 (2015) 1-4. https://doi.org/10.5565/rev/da.263

- GIMÉNEZ-CANDELA, M., CERSOSIMO, R., La enseñanza del derecho animal (Valencia 2021)

- GISIE, L., Comentario jurídico de la Ley 7/2020, de 31 de agosto, de Bienestar, Protección y Defensa de los Animales de Castilla-La Mancha. [2020/6154] - Diario Oficial de Castilla-La Mancha de 07-09-2020, en dA. Derecho Animal (Forum of Animal Law Studies) 12/1 (2021). - DOI https://doi.org/10.5565/rev/da.556

- JIMÉNEZ-LÓPEZ, I, LORENTE RIVERA, C.J., Comentario sobre la nueva ordenanza municipal de protección, tenencia y bienestar animal de Sabadell, en dA. Derecho Animal (Forum of Animal Law Studies) 9/4 (2018) 129-137. https://doi.org/10.5565/rev/da.380

- MARGUÉNAUD, J.-P., L'animal en droit privé (Paris 1992)

- MARGUÉNAUD, J.P. Avant-Propos, en RSDA 1 (2009) 7ss.

- MARGUÉNAUD, J.P., L'entrée en vigueur de “l'amendement Glavany”: un grand pas de plus vers la personnalité juridique des animaux, RSDA 2 (2014), 15ss.

- MARGUÉNAUD, J.-P., La création d'un premier diplôme universitaire de droit animalier en France, en RSDA 1 (2016) 15-24

- MARGUÉNAUD, J.-P., LEROY, J., BOISSEAU-SOWINSKI, L., Code de l'Animal (Paris 2018)

- MARGUÉNAUD, J.-P., BURGAT, F., LEROY, J., Le droit animalier (Paris 2016)

- MERRYMAN, J.H., The civil Law Tradition. An Introduction to the legal Systems of Europe and Latin America (Stanford University Press. ${ }^{\text {rd }}$. Ed. 2007)

- RATHBONE, D., Rural Economy and Society in Roman Italy (Cambridge University Press 2004)

- ROGEL VIDE, C., Los animales en el Código Civil (Madrid 2017)

- VAQUER ALOY, A., El derecho civil catalán: presente y futuro, en Revista jurídica de Navarra 46 (2008) 69ss. http://hdl.handle.net/10459.1/66188

\section{FUENTES}

- Amendement n. 59 du 11 avril 2014 presente par M. Glavany et al. : https://www.assembleenationale.fr/14/amendements/1808/AN/59.asp

- Art. 511-1.3 CCCat.: https://www.boe.es/eli/es-ct/1/2006/05/10/5/con

- Art. 515-14 C.civ.: https://www.legifrance.gouv.fr/codes/article_lc/LEGIARTI000030250342/

- Art. 641a ZGB: https://www.fedlex.admin.ch/eli/cc/24/233_245_233/de

- Ley 13/1984, de 20 de marzo, de reforma de la Compilación del derecho civil de 1960 https://www.boe.es/eli/es-ct/1/1984/03/20/13

- Ley $3 / 1988$, de 4 de marzo, de protección de los animales https://www.boe.es/eli/es$\mathrm{ct} / 1 / 1988 / 03 / 04 / 3$

- Ley 5/2006, de 10 de mayo, del libro quinto del Código civil de Cataluña, relativo a los derechos reales «DOGC» núm. 4640, de 24/05/2006, «BOE» núm. 148, de 22/06/2006, BOE-A-2006-11130

- Ley de protección de los animales de Cataluña https://www.boe.es/buscar/pdf/2008/DOGC-f-200890016-consolidado.pdf

- Ley 7/2020, de 31 de agosto, de Bienestar, Protección y Defensa de los Animales de Castilla-La Mancha https://www.boe.es/eli/es-cm/1/2020/08/31/7

- TFUE, art.13 https://eur-lex.europa.eu/legal-content/ES/TXT/?uri=CELEX:12016E013 
- \$285a ABGB: https://www.ris.bka.gv.at/eli/jgs/1811/946/P285a/NOR12018011

- $\$ 90 a$ BGB: https://www.gesetze-im-internet.de/bgb/_90a.html
ANEXO I
Normativa sobre fauna urbana en Cataluña
(jabalís, cotorras, palomas, colonias de gatos ferales)

\section{Normativa en común:}

Nivel internacional:

- Convenio Europeo sobre protección de animales de compañía, hecho en Estrasburgo el 13 de noviembre de 1987, ratificado por España, en el BOE núm. 245, de 11 de octubre de 2017, páginas 98971 a 98982, con fecha de efecto desde el 1 de febrero de 2018. - Instrumento de ratificación del Convenio Europeo sobre protección de animales de compañía, hecho en Estrasburgo el 13 de noviembre de 1987. - https://boe.es/diario_boe/txt.php?id=BOE-A-2017-11637

- Convenio de las Naciones Unidas sobre Diversidad Biológica, 1992, Río de Janeiro (Brasil), ratificado por España en el BOE núm. 27, de 1 de febrero de 1994, páginas 3113 a 3125 https://www.boe.es/buscar/doc.php?id=BOE-A-1994-2193

Nivel europeo:

- Directiva de Aves - Directiva 2009/147/CE del Parlamento Europeo y del Consejo, de 30 de noviembre de 2009, relativa a la conservación de las aves silvestres - https://eur-lex.europa.eu/legalcontent/ES/TXT/?uri=celex\%3A32009L0147

- Directiva de Hábitats - Directiva 92/43/CEE del Consejo, de 21 de mayo de 1992, relativa a la conservación de los hábitats naturales y de la fauna y flora silvestres https://www.boe.es/buscar/doc.php?id=DOUE-L-1992-81200

Nivel nacional:

- Constitución Española, BOE, 29 de diciembre de 1978, núm. 311: artículos 45, 148.1.9, 149.1.23 https://www.boe.es/eli/es/c/1978/12/27/(1)

- Ley 7/1985, de 2 de abril, Reguladora de las Bases del Régimen Local: art. 25.2, 27.3 https://www.boe.es/buscar/act.php?id=BOE-A-1985-5392

- Ley estatal 42/2007, de 13 de diciembre, del patrimonio natural y de la biodiversidad: art 54 y 61 https://www.boe.es/buscar/act.php?id=BOE-A-2007-21490

- Ley 8/2003, de 24 de abril, de sanidad animal. BOE $n^{\circ} 99$, de 25 de abril de 2003 https://www.boe.es/buscar/act.php?id=BOE-A-2003-8510

Nivel autonómico:

- Ley 22/2003, de 4 de julio, de protección de los animales, BOE, núm. 189, de 8 de agosto de 2003, 30724 a 30738 - https://www.boe.es/buscar/doc.php?id=BOE-A-2003-15900

- Decreto legislativo 2/2008, por el que se aprueba el Texto refundido de la Ley de protección de los animales, DOGC, núm. 5113, de 17 de abril de 2008, 29665 a 29697: artt. 2, 9, 16, 33.1, 35 https://www.boe.es/buscar/doc.php?id=DOGC-f-2008-90016

Nivel municipal: 
- Ordenanza de protección, tenencia y venta de animales en Barcelona aprobada por el Ayuntamiento Pleno el 25 de julio de 2014: artículos 10,33 https://ajuntament.barcelona.cat/benestaranimal/es/legislacion-y-normativa

Normativa penal:

- Ley Orgánica 10/1995 de 23 de noviembre, Código penal: artículos 336 (delito por uso de veneno o método de caza no autorizado con eficacia destructiva o no selectiva) https://www.boe.es/buscar/act.php?id=BOE-A-1995-25444

\section{Normativa adicional específica:}

\section{Cotorras:}

- Reglamento (UE) n ${ }^{\circ}$ 1143/2014 del Parlamento Europeo y del Consejo, de 22 de octubre de 2014, sobre la prevención y la gestión de la introducción y propagación de especies exóticas invasoras https://eur-lex.europa.eu/legal-content/ES/TXT/?uri=CELEX\%3A32014R1143

- Ley 42/2007, de 13 de diciembre, del Patrimonio Natural y de la Biodiversidad (supra): art. 3.13, $54,64$.

- Real Decreto 630/2013, de 2 de agosto, por el que se regula el Catálogo Español de Especies Exóticas Invasoras - https://www.boe.es/buscar/act.php?id=BOE-A-2013-8565

\section{Colonias felinas:}

- Decreto Legislativo 2/2008 de Cataluña (supra): art 3,11, 16, 19

- Orden sobre la protección, la tinença y la venta de animales de barcelona, 2014 (supra): art. 2.9, 28.

- Ley Orgánica 10/1995 de 23 de noviembre, Código penal (supra): artículos 336 (delito por uso de veneno o método de caza no autorizado con eficacia destructiva o no selectiva), 337 (delito de maltrato animal) y 337 bis (delito de abandono)

- Programa GIF-VET, noviembre de 2018, el Consejo de Colegios de Veterinarios de Catalunya (CCVC) - https://www.colvet.es/sites/default/files/2019-06/DOSSIER\%20GIF-VET.pdf

- Norma UNE 313001:2016 "Centros de protección animal y residencias de animales de compañía. Gestión sanitaria y bienestar animal” - https://tienda.aenor.com/norma-une-313001-2016-n0057546

Jabalís:

- Real Decreto 1082/2009, de 3 de julio, por el que se establecen los requisitos de sanidad animal para el movimiento de animales de explotaciones cinegéticas, de acuicultura continental y de núcleos zoológicos, así como de animales de fauna silvestre - https://www.boe.es/buscar/doc.php?id=BOEA-2009-12206

- Resolució ARP/1015/2020, de 12 de maig, per la qual es fixen les espècies objecte d'aprofitament cinegètic, els períodes hàbils de caça i les vedes especials per a la temporada 2020-2021 en tot el territori de Catalunya. Diari Oficial de la Generalitat de Catalunya $n^{\circ} 8134$, de 15 de maig de 2020 $-$ 2020_2021.pdf

https://www.fccterresebre.cat/wp-content/uploads/2020/05/Ordre-de-vedes-temporada-

- Decisión de Ejecución(UE) 2021/544 de la Comisión de 25 de marzo de 2021 por la que se modifica el anexo de la Decisión de Ejecución 2014/709/UE, sobre medidas de control zoosanitarias relativas a la peste porcina africana en determinados Estados miembros [notificada con el número C(2021) 2107] - https://eur-lex.europa.eu/legal-content/ES/TXT/?uri=CELEX:32021D0544

- Programa de seguiment de les poblacions de senglar a Catalunya. Temporada 2019-2020 http://agricultura.gencat.cat/web/.content/06-medi-natural/caca/enllacos-documents/informestecnics/programa-seguiment-poblacions-senglar-sus-scrofa/fitxersbinaris/seguiment_senglar_cat_2019-20-WEB.pdf 